\title{
Segunda reunión plenaria del Grupo Latinoamericano de Instituciones del Cemento y del Concreto (GLAICYC)
}

\author{
Río de Janeiro, Brasil, 15-17 de octubre 1979
}

Prof. Dr. J. CALLEJA

Vicedirector del IETCC

MADRID (España)

\section{INTRODUCCION Y PROGRAMA}

En los días 15 al 17 de Octubre de 1979 tuvo lugar en Río de Janeiro la 2. ${ }^{a}$ Reunión Plenaria del GLAICYC (Grupo Latinoamericano de Instituciones del Cemento y del Concreto), del que forman parte las siguientes Asociaciones, Institutos y Países:

1. Associaçao Brasileira de Cimento Portland (ABPC).

2. Asociación Centroamericana del Cemento y Concreto (ACCC), representada por el momento por Panamá.

3. Asociación Uruguaya de Fabricantes de Cemento Portland (AUFCP).

4. Asociación Venezolana de Productores de Cementos (AVPC).

5. Instituto del Cemento Portland Argentino (ICPA).

6. Instituto Colombiano de Productores de Cemento (ICPC).

7. Instituto Chileno del Cemento (IChC).

8. Instituto Ecuatoriano de Productores de Cemento (IEPC).

9. Instituto Eduardo Torroja de la Construcción y del Cemento (IETCC).

10. Instituto Paraguayo del Cemento Portland (IPCP).

La primera Reunión Plenaria (fundacional) del GLAIC,YC tuvo lugar en Medellín (Colombia), en Septiembre de 1973, constituyéndose la Secretaría General del Grupo en la sede del ICPC, con carácter permanente hasta la 2. ${ }^{a}$ Reunión del Grupo, y recayendo el cargo 
de Secretario General en la persona del Ing. Jorge Ignacio PAZ PARRA. De esta reunión se dió cuenta en su momento y lugar (Materiales de Construcción - IETCC - núm. 169, Enero-Febrero-Marzo 1978).

A la Segunda Reunión Plenaria de Río de Janeiro asistieron representantes de los siguientes países iberoamericanos, así como del organismo internacional europeo CEMBUREAU:

Por ARGENTINA

Por BRASIL

Por CEMBUREAU

Por CHILE

Por COLOMBIA

Por ESPAÑA

Por PANAMA

Por PARAGUAY

Por URUGUAY

Totales: $8-1$
4. Eduardo D. CASH.

Carlos E. DUVOY.

Hector D. FIERRO.

Dante J. E. VERONELLI.

7. Francisco de Assis BASILIO.

Rawilsean DUTRA DE ALMEIDA L.

Salvador E. GIAMUSSO.

Airton GRILL.

Antonio KROPF SOARES.

Argos MENNA BARRETO.

Carlos A. THOMAZ.

3. H. C. COLLIS.

Harald DYCKEERHOFF.

Ernesto GARAU Ll.

1. Eduardo GOMIEN D.

2. Germán BOTERO A. Jorge I. PAZ P.

3. Francisco ARREDONDO V. José CALLEJA C. Ernesto GARAU Ll.

1. Santiago TORRIJOS.

2. Ramón CENTURION N. Higinio VILLALVA.

4. José L. AMO.

Darwin ARTIGALAS.

Oscar R. PAROLI.

Gustavo VAZQUEZ F.

27

Hizo las veces de anfitrión el miembro brasileño del Grupo: la ABCP.

La Reunión se desarrolló con arreglo al siguiente programa y temario de sesiones técnicas y económicas:

\section{Día 14 de octubre}

noche: cocktail de recepción en el Hotel Gloria sede de la Reunión. 


\section{Día 15 de octubre}

8.30 a $9 \mathrm{~h}$ Inscripciones.

9.00 a 10 h Acta de Medellín. Informe sobre el desarrollo de las conclusiones y recomendaciones, por el Ing. Jorge I. PAZ.

10.00 a $11 \mathrm{~h}$ Economía de combustibles derivados del petróleo: Estructura del consumo de combustibles en la industria brasileña del cemento, por el Ing. Argos MENNA.

11.00 a $12 \mathrm{~h}$ Consideraciones sobre la economía de combustibles y otros derivados del petróleo en la fabricación y en la utilización de los cementos, por e]. Prof. Dr. José CALLEJA.

14.00 a $15 \mathrm{~h}$ Promoción directa del empleo del hormigón, por el Ing. Salvador E. GIAMUSSO.

15.00 a 16 h Promoción (indirecta) del empleo del hormigón: cursos y actividades a través de asociaciones, por el Ing. Carlos A. THOMAZ.

16.00 a $17 \mathrm{~h}$ Comercio Zonal e Internacional en los países latinoamericanos por el Ing. Argos MENNA.

\section{Día 16 de octubre}

9.00 a 10 h Normalización COPANT e ISO. Sello de Conformidad, por el Ing. Francisco de Assis BASILIO.

10.00 a $11 \mathrm{~h}$ Automatización y economía de combustibles, por el Prof. Dr. José CALLEJA. (Introductor del tema y conductor del Coloquio).

11.00 a 12 h Investigación Tecnológica y Científica, por el Ing. Francisco de Assis BASILIO.

14.00 a 16 h Producción de cemento en América Latina. Datos estadísticos y Proyección. Control de precios y sus consecuencias.

16.00 a $17 \mathrm{~h}$ Cuestiones pendientes. Conclusiones y Clausura.

\section{Día 17 de octubre}

$8.00 \mathrm{~h} \quad$ Visitas técnicas y turísticas.

Como es bien patente, las sesiones técnicas se centraron sobre cuatro puntos fundamentales: energía, promoción, normalización e investigación, mientras que las económicas versaron acerca de: producción, comercio y precios.

Cada tema tratado dió lugar a interesantes intervenciones que, según las circunstancias, revistieron el carácter de "ruedas de mesa" o de coloquios libres y espontáneos.

La Presidencia de la Reunión le fue amablemente ofrecida al miembro español del Grupo, en la persona del Ing. Francisco ARREDONDO, Director del IETCC, el cual la aceptó agradecido. 


\section{Desarrollo Del PRograma}

\subsection{Acta de Medellín}

\section{Ing. Jorge I. PAZ}

Como continuación del Acta de la Reunión de Medellín la Secretaría General del GLAICYC presentó un Informe de 7 páginas, con una Introducción, 10 puntos y 2 conclusionesrecomendaciones, firmado por el Secretario, Ing. Jorge I. PAZ.

En los puntos del Informe se trataron aspectos de la organización y funcionamiento del Grupo en los 6 años de su actuación y se expusieron las principales actividades desarro. lladas por el mismo, así como la creación e ingreso en él de nuevas asociaciones nacionales, como la uruguaya, y de nuevos institutos, como el ecuatoriano y el paraguayo.

Entre las actividades del Grupo se detallaron la reunión de la Subregión Norte en Caracas y 1974, las tres de la Subregión Sur en Sao Paulo 1974, Montevideo 1977 y Santiago 1978, así como la de Coordinación en Caracas y 1975, con diversas asistencias y participaciones.

Materias tratadas en estas reuniones, e informes presentados en las mismas, fueron, entre otros, los relativos a actividades, experiencias y programas conjuntos para llevar a cabo en temas como el suelo-cemento y su promoción; la tecnología del hormigón; el hormigón preamasado; los tipos especiales de hormigón; las presas de hormigón; la microscopía del clínker; las normas nacionales en relación con la calidad del cemento, su variabilidad y su control estadístico, así como el sello de calidad; la participación en la RILEM y en el COPANT (Comité Panamericano de Normas Técnicas); la normalización de los áridos y del propio hormigón; las estadísticas y los viajes y visitas técnicas.

En cuanto a las relaciones de la Secretaría General con las Instituciones del Grupo y otras instituciones, el informe trató de los 12 números editados del Boletín Informativo y de las visitas efectuadas y recibidas por la Secretaría General: 10 de las primeras, entre las que destacan las realizadas a la ACCC y al IChC, y 8 de las segundas; aparte de las visitas de observación y/o estudio realizadas por profesionales de otras Instituciones a las del Grupo.

Asimismo trató el Informe de la cooperación mantenida por la Secretaría General del Grupo con la ACI, el CEMBUREAU, el CEB, OFICEMEN y la PCA, así como con el IMCYC, con la CIH (Confederación Iberoamericana del Hormigón Preparado), el IDCC (Instituto Dominicano del Cemento y del Concreto) y la ATIC (Asociación Técnica de la Industria del Cemento - de Portugal-). Estas dos últimas entidades fueron invitadas a participar en la 2." Reunión del Grupo en Río de Janeiro y 1979. En cuanto a la CIH, el Informe mencionó sus dos Congresos llevados a cabo, el primero en Zaragoza (España) en 1976 y el segundo en Méjico en 1978.

Con relación al intercambio de publicaciones, el Informe hizo destacar el cumplimiento del compromiso y la diligencia puesta en el mismo por parte de las asociaciones y, entre otras por el IETCC.

Respecto de la cooperación tecnológica, el Informe mencionó: el entrenamiento de técnicos de unos países en otros en materias como microscopía del clínker y tecnología del cemento y del hormigón, entre varios más, con activa participación española del IETCC en dicha labor; y las conferencias, cursos, simposios y seminarios desarrollados por las asociaciones miembros del Grupo, destacando en tal sentido la actuación española del IETCC en Argentina, Brasil, Centroamérica, Colombia, Chile, República Dominicana, Uruguay y Venezuela.

8 
En cuanto a la labor de capacitación de técnicos de la industria del cemento, el informe subrayó las actividades de Brasil, Colombia y España, a través de las Reuniones (semestrales) de Técnicos del Cemento de Brasil, de las (dos) Jornadas Colombianas del Cemento, y de los VI Coloquios de Directores y Técnicos de Fábricas de Cemento de España desarrollados en 1976 con asistencia y participación colombiana. Asimismo se mencionó en el Informe el Curso Holderbank de Cemento para todos los países latinoamericanos, dado en Bogotá y julio de 1975.

Después de mencionar las tareas sobre intercomunicación de actividades por parte de las Instituciones, sobre traducciones, sobre normalización COPANT (Comisión Panamericana de Normas Técnicas, a la que hasta hace poco tiempo pertenecían los EE.UU.) y sobre recopilación de estadísticas sobre cemento por parte de la Secretaría General del Grupo, el Informe terminó con la conclusión de la labor positiva realizada por el GLAICYC, y con la recomendación de incrementar la comunicación y cooperación entre las instituciones del Grupo, para lo cual se proponía el nombramiento de coordinadores regionales como delegados permanentes del Secretario General del mismo.

Después de un debate sobre la necesidad de incrementar la cooperación y la creación de los coordinadores regionales, tema éste que quedó aplazado y pendiente de resolución, el Informe fue aprobado por unanimidad con general expresión de agradecimiento y felicitación a su redactor, el Ing. Jorge I. PAZ.

\subsection{Estructura del consumo de combustibles en la Industria Brasileña del Cemento}

\section{Ing. Argos MENNA (Brasil)}

En la exposición del tema su presentador mencionó las encuestas realizadas y los cuestionarios preparados por el CNP (Consejo Nacional del Petróleo) en demanda de datos a la industria brasileña en general, sobre consumo de derivados del petróleo. La industria del cemento respondió con interés y entusiasmo.

Entre las medidas drásticas para la reducción del consumo de combustible en la fabricación del clínker mencionó la eliminación de la vía húmeda y su sustitución por la vía semiseca, llevada a cabo por alguno de sus varios procedimientos. Entre las medidas discretas citó el mayor y mejor control de la temperatura de los gases a la salida del horno, la evitación del aire parásito, el mejor intercambio de calor y la mayor recuperación y aprovechamiento de éste.

Como medidas complementarias de otro orden - y de primera magnitud- habló de la sustitución de clínker por adiciones activas (escorias, cenizas volantes y puzolanas artificiales -arcillas térmicamente activadas-) para la fabricación de cementos siderúrgicos y puzolánicos. En tal sentido mencionó, de acuerdo con las normas brasileñas, los cementos de alto horno AF con 25-65 \% de escorias y los puzolánicos POZ con 10-40\% y 10-30 \% de puzolanas naturales, artificiales o cenizas volantes, así como los cementos portland comunes $\mathrm{u}$ ordinarios $\mathrm{CPC}$ con $10 \%$ de estas adiciones.

Pasó después a tratar de los consumos específicos de calor en las cementerías de diversos países, así como de los combustibles y de sus potencias caloríficas inferiores en kcal/ $/ \mathrm{kg}$, referidas al fuel-oil (9.500-9.800), al carbón vegetal (5.000) y al carbón mineral pobre con $35-40 \%$ de cenizas y no más de $2 \%$ de azufre (4.700). A propósito de todo ello el 
autor se extendió en detalles sobre un programa de carbón para cementería en el Brasil, con objeto de sustituir de forma total, po de carbón mineral.

En el coloquio que suscitó la exposición del tema se solicitaron ideas para el paso del empleo de fuel-oil al del carbón; se habló de las pocas ventajas de dicho cambio $y_{\text {, de sus }}$ dificultades, sobre todo cuando no se dispone de carbones aceptables y de calidad suficientemente homogénea; se mencionó la necesidad, en tales casos, de proceder a mezclas previas; se citó la tendencia al alza del precio del carbón, como consecuencia del aumento de su demanda; se trató de la precisión de importar carbón, así como de su redistribución, suministro y transporte, por parte de algunos países con condiciones precarias al respecto, por escasez y/o por carestía, así como por falta de calidad de sus propios carbones. Y como solución general y prácticamente unánime se consideró la del empleo de adicio. nes en sustitución de clínker, arbitrio que en ocasiones reviste un interés político además del técnico, y en cuya aplicación algún país se ha esforzado con la ayuda de otro mediante el desarrollo de cursos sobre adiciones y materiales puzolánicos para cementos, dirigidos a sus técnicos cementeros.

En cuanto a la posición europea al respecto, se manifestó que no era -ni podía serclara y unitaria, por cuanto que las tendencias eran muy variables según los países, en función de sus diferentes disponibilidades o facilidades de acceso a los mercados de fueloil, gas natural o carbón; pero que, en todo caso y en general, la inclinación hacia una vuelta al empleo del carbón no era fuerte.

\subsection{Consideraciones sobre la economía de combustibles y otros derivados del petróleo en la fabricación y en la utilización de los cementos}

\section{Prof. Dr. José CALLEJA (España)}

Comenzó el autor ofreciendo sus disculpas por su espontaneidad al tratar, con su aportación, de relacionar puntos aislados del temario de la reunión, entre sí y con el que parece encabezar el programa de la misma: la economía de combustibles derivados del petróleo, dentro de las industrias de la fabricación del cemento. Habló a título personal de la postura y situación españolas frente a algunos aspectos del tema.

Mencionó el interés de la Agencia Internacional de la Energía acerca del mismo y recordó los puntos de vista norteamericanos expuestos al respecto en la 1. ${ }^{a}$ Reunión del GLAICYC en Medellín (Colombia) y Diciembre de 1973. Entre tales puntos de vista destacó el regreso al empleo del carbón en los EE.UU., como una de las alternativas posibles - la otra era (y es) la importación de clínker y/o cemento-, ante la reducción del suministro de gas natural a la industria cementera norteamericana, el aumento general del precio de los combustibles, la no atenuación de las medidas contra la contaminación durante el período de crisis energética, y la inmovilización del precio del cemento, atentatoria contra una razonable rentabilidad del mismo.

Precisó el autor la posible utilización del carbón, no directamente como combustible, sino como fuente de combustible gaseoso por hidrogenación previa, dado el valor energético de los productos que se pueden obtener del carbón, y de las grandes reservas mundiales de este mineral comparadas con las del petróleo.

Se manifestó escéptico en cuanto a la posibilidad de utilizar, con tales fines, o bien directamente, carbones de baja y heterogénea calidad, con contenidos altos de cenizas y/o 
azufre, proclives a crear problemas técnicos en la maquinaria - horno- y en el proceso -clinkerización-, lesivos para la producción y la calidad, así como para el costo del proceso y del producto.

Análogo escepticismo mostró en cuanto a las posibilidades de la automatización, conveniente y necesaria en otros muchos aspectos, para producir ahorros de combustible. Tampoco manifestó esperanza de que se consiguiesen análogos objetivos, a muy corto plazo, por el desarrollo de nuevos tipos de cementos o de procesos fundamentados en estudios básicos sobre la termodinámica del horno o la físico-química del clínker, si bien la investigación científica y sobre todo técnica en tales aspectos era indispensable, tanto para la creación de nuevo conocimiento como para el mejor aprovechamiento del ya adquirido; añadió que dicha investigación podía y debía ser fomentada y progresar en tal sentido.

Por todo ello pasó a exponer la solución alternativa general de los cementos con adiciones, así como la particular de España, contemplada en sus actuales normas vigentes para cementos. A tales efectos justificó cuantitativamente el ahorro de combustible y calorías en clinkerización, e hizo consideraciones sobre la incidencia de las adiciones en el consumo total de kilowatios-hora en la molienda.

Salió al paso de posibles argumentos sofisticados, basados en la mayor lentitud de endurecimiento de los cementos con adiciones respecto de los portland sin ellas, argumentos que invocan unos plazos mayores para el desencofrado y una mayor duración de las obras -o el empleo de dosificaciones mayores para desencofrar en el mismo plazo-, a fin de desvirtuar así el ahorro. Hay que entender — dijo el autor-que este ahorro debe ser y es de energía de combustible derivado del petróleo, que el país que lo importa — si puede- tiene que pagar en divisas — si las tiene-; mientras que el mayor costo por una duración algo más prolongada de las obras es en concepto de mano de obra, la cual se paga siempre en moneda nacional. Que el constructor aparentemente lleve en ello peor parte que el fabricante de cemento es algo accidental —añadió-, puesto que sucederá a la inversa en cuanto el fabricante se vea obligado a utilizar carbón y a mantener una determinada calidad de cemento a un mismo precio - lo cual es siempre muy difícil y pocas veces posible-.

El autor señaló la conveniencia de ensayar previamente, en hormigones reales, cualquier nuevo cemento con adiciones que se fabrique, así como de especificarlo adecuadamente en las normas, garantizando por diversos medios su calidad y la constancia de la misma. Puso al respecto ejemplos de cómo hacerlo para eliminar las posibles grandes dispersiones que las normas vigentes en la mayoría de los países no son capaces de evitar, y para eludir problemas en las obras.

Pasó a tratar de la promoción del hormigón y del cemento en las obras públicas — sobre todo de carácter vial- y en la construcción y en la edificación industrializada (prefabricada) - preferentemente de carácter social-, e hizo consideraciones sobre la posible competitividad de ciertos derivados del cemento con los equivalentes derivados de los plásticos, así como de los firmes blancos y rígidos de hormigón con los firmes negros y flexibles de asfalto. En relación con ello expuso nuevas posibilidades de ahorro de derivados del petróleo en favor del cemento. Citó asimismo algunas nuevas aplicaciones no tradicionales de este material.

Finalmente, indicó la necesidad de un mayor acercamiento y compenetración entre los profesionales técnicos de la construcción y de la fabricación del cemento, a base de un mayor conocimiento de las técnicas de los unos por parte de los otros, a fin de conseguir entre 
ambos una mayor eficacia en la utilización y en el ahorro de los recursos energéticos naturales, caros y escasos, así como de los cementos con adiciones, sin problemas para el hormigón.

\subsection{Promoción (directa) del empleo del hormigón}

\section{Ing. Salvador E. GIAMUSSO (Brasil)}

Presentó el ponente un organigrama de la $\mathrm{ABCP}$ en el que, aparte de la Dirección, del Grupo de Trabajo sobre Ingeniería de la Calidad, y de las Divisiones Administrativa y de Tecnología del Cemento, figuran la de Promoción Técnica, encabezada por el autor y encargada de la promoción llamada directa, y la de Enseñanza y Divulgación, dirigida por el Ing. C. A. THOMAZ y responsable de la llamada promoción indirecta.

La División de Promoción Técnica (directa) tiene departamentos en diversas ciudades importantes de las zonas norte, centro y sur del Brasil, siendo el jefe de esta última el propio Ing. S. E. GIAMUSSO.

La promoción directa — dijo el autor - tiene como base la calidad y la propaganda del hormigón, expuesta la primera y desplegada la segunda mediante contactos realizados a base de mesas redondas, asociaciones, concursos, conferencias, congresos, etc., así como por medio de visitas a otras instituciones. Particular relación -añadió- existe y se mantiene y acrecienta a tales efectos con técnicos de centrales hormigoneras y con otros encargados de la elaboración de normas técnicas para hormigón y sus materiales.

Entre las líneas de promoción citadas por el autor figuró en primer lugar la de los pavimentos de hormigón, en los que este material resulta competitivo y favorecido en el aspecto del precio, aparte problemas de maquinaria y equipo. Por otra parte, la economía de iluminación favorable al firme blanco es evidente.

Otra línea de promoción citada fue la del suelo-cemento. Existe en contra de la normal promoción del cemento en esta línea — dijo el expositor- la decisión unilateral y arbitraria de algunos contratistas en el sentido de rebajar sin más las dosificaciones de cemento, arguyendo a veces una mejor calidad de éste, con evidente desconocimiento de causa, al cual sería necesario combatır. Una forma de hacerlo — dijo- es la de desarrollar cursos para encargados de obra, en los cuales se ponga de manifiesto la improcedencia técnica de tales decisiones.

Otras posibles líneas de promoción explotables citadas por el autor, fueron la difusión del empleo del hormigón, del suelo-cemento y del cemento en general en medios rurales, en cuanto a silos, depósitos, pavimentos, etc.; la utilización de los citados materiales en vías residenciales de poco tránsito; y la fabricación de elementos monolíticos con cemento, tales como losas, bloques, bovedillas, tejas, etc.

Terminada la exposición se desarrolló un coloquio que tomó el carácter de "rueda de mesa", en la que sucesivamente las distintas delegaciones fueron exponiendo sus líneas de promoción del cemento y del hormigón.

Así, por parte argentina se indicó que lo expuesto por el ponente era válido para el ICPA, según consta en su memoria de 1978. El Ing. C. E. DUVOY expuso la estructura y organización del ICPA en Secciones (técnicas centralizadas) y Seccionales (dispersas por el país), encargadas respectivamente de la investigación en laboratorios de ensayo de un 
nuevo edificio, y de la promoción a través del desarrollo de pavimentos, estructuras y hormigón pretensado, y del uso del cemento en agricultura, etc., en tanto que promoción directa; así como de exposiciones, cursillos, asistencia a actos y asociaciones internacionales sobre hormigón pretensado (FIP) y hormigón preamasado, o nacionales relativos a tecnología industrial y normalización (INTI, IRAM). En cuanto a labor de investigación y desarrollo tecnológico - dijo-, el ICPA también hace promoción con asistencia técnica gratuita a los usuarios del cemento, con demostraciones y estudios técnico-económicos sobre traviesas, postes, tramos, etc., de hormigón, y con trabajos sobre utilización de fibras y áridos volcánicos, y cáscara de arroz y áridos ligeros para hormigón coloidal; y también con el aprovechamiento de los desechos de hormigón en el proyecto llamado "Econocret". Las publicaciones del ICPA - terminó diciendo- contribuyen a la promoción del hormigón por su efecto multiplicador.

En el caso del Paraguay el Dr. CENTURION habló de la creación del nuevo Instituto Paraguayo del Cemento Portland (IPCP) y de su preocupación por la promoción del uso del cemento en vialidad, a causa de la crisis del petróleo y sus derivados.

Análogamente, en el caso del Uruguay el Ing. O. R. PAROLI informó sobre el nacimiento de la Asociación Uruguaya de Fabricantes de Cemento Portland (AUFACEP) y del inicio de la promoción del cemento a base de cursillos y conferencias, con el apoyo del Departamento Técnico del Hormigón dentro de la Asociación y su proyección en los campos de la vialidad y del suelo-cemento.

De una promoción similar del cemento en el último de los mencionados campos y en el ámbito rural habló el Ing. S. TORRIJOS por parte de Panamá, en lo que se refiere a la ACCC.

Por Colombia informó el Ing. G. BOTERO, el cual habló de una promoción cualitativa por parte del ICPC, encaminada no sólo a un mayor, sino también a un mejor consumo de cemento y hormigón. Promoción tanto más necesaria — dijo-, por cuanto que el país tiene petróleo y asfalto y por otra parte aumenta la producción de cemento. Citó como líneas de promoción el desarrollo del suelo-cemento y el empleo del cemento en adoquines para pavimentaciones, así como para pavimentos viales de aeropuertos dada la naturaleza del suelo y la magnitud de las cargas de transporte. La promoción del cemento -añadió- es un trabajo a largo plazo en manos de los actuales estudiantes de ingeniería. Por otra parte -concluyó- la promoción indirecta la realiza el ICPC a través de las relaciones con otras instituciones, y en particular con las dedicadas a normalización.

El Ing. E. GOMIEN habló del Instituto Chileno del Cemento -y hoy también del Hormigón- como de una entidad vuelta hacia el usuario para que éste utilice más cemento y en mejores condiciones técnicas. Actúa, pues, —dijo-, de fábricas hacia afuera, ocupándose de las ventas, pero no de la producción, ni de los precios, ni de la distribución, ni siquiera de las estadísticas. Mencionó la producción, repartida y equilibrada, así como las circunstancias y vicisitudes de las fábricas del país, y la vinculación de una de ellas al Grupo Blue Circle británico y de otra al Grupo suizo Holderbank. Informó sobre la promoción directa del empleo de cemento en obras públicas (vialidad y presas hidroeléctricas), así como en suelo-cemento, propugnando el mayor y mejor empleo del mismo. Hizo referencia también a los problemas del control en la tecnología del hormigón y a los varios informes técnicos aportados por Brasil acerca de los trabajos realizados y en marcha en los campos de la vialidad y de las presas. Citó finalmente como posibles líneas futuras de promoción la creación de becas, la organización de cursos y la paticipación en organización de cursos y la participación en 
Por parte de España el Ing. F. ARREDONDO puso como ejemplo de promoción moderna y original del empleo del cemento, la construcción de barcos de hormigón, y en particular la de barcos metaneros para el transporte de metano licuado a temperaturas inferiores a $-160^{\circ} \mathrm{C}$. Este tema ya había sido expuesto in extenso con anterioridad por el Ing. A. PAEZ en una ponencia titulada: "Nuevos usos del cemento: Barcos de hormigón", presentada en el Grupo de Sesiones Técnicas dedicadas a la Utilización del Cemento, en los VI Coloquios de Directores y Técnicos de Fábricas de Cemento, organizados por el IETCC y celebrados en su sede de Madrid, en 1976.

Bien en aquella exposición o bien en el coloquio al que la misma dio ilugar entonces, y en el que, entre otros participaron el Ing. ARREDONDO y el Dr. CALLEJA, se tocaron temas que fueron recordados por el Ing. ARREDONDO en la aportación presentada en esta ocasión al GLAICYC.

Algunos de los temas recordados fueron: la necesidad de una pantalla de acero especial inserta en el hormigón para evitar la penetración del líquido a través de éste; la necesidad de aligerar el pretensado a fin de reducir peso y disminuir la desfavorable relación de masas entre barcos de hormigón y barcos de acero equivalentes, para lo cual se deben utilizar áridos ligeros (mejor semipesados) de alta resistencia; la necesidad de conseguir hormigones de muy alta resistencia con muy baja relación agua/cemento mediante el empleo de superplastificantes, etc.

Por parte del CEMBUREAU el Sr. COLLIS dijo que en el seno de dicha Asociación apenas se llevaba a cabo promoción alguna, si bien ésta se realizaba individualmente por los miembros del CEMBUREAU, en una u otra medida. Citó como líneas europeas de promoción en este sentido la construcción de viviendas unifamiliares, el empleo del cemento y del hormigón en la agricultura (obras de regadío), y la propaganda del material entre los pequeños consumidores, cuya capacldad de consumo va en aumento. A todo ello -añadió- contribuye la propia imagen del hormigón que el CEMBUREAU, tal vez como promoción indirecta, se encarga de hacer llegar a sus miembros y al público en general, a través de la publicación de sus Technical Letters, News Letters, y otras de carácter más específico y detallado, según las circunstancias.

\subsection{Promoción (indirecta) del empleo del hormigón}

\section{Ing. Carlos A. THOMAZ (Brasil)}

El expositor del tema comenzó haciendo un bosquejo histórico de la $\mathrm{ABCP}$, en cuyo organigrama figura encabezando la División de Enseñanza y Divulgación, una de cuyas misiones principales es precisamente la promoción indirecta del empleo del cemento y del hormigón. Esta promoción - dijo- se lleva a cabo desde 1941, fecha en que la ABCP comenzó sus actividades, a través de la realización de cursos, de la publicación de libros y de la elaboración de recomendaciones y normas, estas últimas de acuerdo con la ABNT (Asociación Brasileña de Normas Técnicas), entidad con la cual colabora la ABCP. Las materias tratadas en las publicaciones con fines de promoción han sido varias, entre las cuales - añadió- cabe mencionar, aparte de las relativas a normalización, las que se refieren a tecnología del hormigón y del suelo-cemento, a desarrollo de la vivienda, etc.; y en cuanto a la realizacion de cursos, cabe citar — dijo- uno llevado a cabo sobre microscopía del clínker para control de fabricación. 
En el coloquio que siguió a la exposición cabe destacar la observación hecha por el Ing. DUVOY acerca de la promoción directa, que como más importante él considera masiva y orientada hacia técnicos, y la indirecta, de carácter individual y —dijo- más polémica a su juicio en cuanto a eficacia y resultados.

\subsection{Comercio zonal e interzonal en los países latinoamericanos}

\section{Ing. Argos MENNA (Brasil)}

Dentro de la parte del programa dedicada a los asuntos económicos el Ing. MENNA desarrolló el tema relativo a relaciones comerciales del cemento en Latinoamérica. Hizo una exposición de los puestos ocupados por los distintos países en la producción anual mundial, haciendo resaltar el destacado décimo lugar - muy próximo al noveno- ocupado por Brasil en la misma, con una cifra que supera los 23 millones de toneladas. Habló después de la distribución de las 57 fábricas en los distintos Departamentos del país, con una mayor concentración en los de Sao Paulo y Minas Gerais. Detalló luego la producción de los distintos tipos de cemento por fábricas y zonas, indicando que 7 fábricas producían cementos AF (de alto horno), y que los cementos puzolánicos se producían en la zona carbonífera del sur del país, aprovechando las cenizas volantes de las centrales termo-eléctricas. Hizo destacar el hecho del gran desarrollo de la producción de cementos siderúrgicos y puzolánicos $-\mathrm{y}$ sobre todo de los primeros- entre 1975 y 1978, hasta el punto de que los cementos siderúrgicos habían pasado al primer puesto de la producción, habiendo ascendido desde un 8 por ciento a un 62 por ciento. Ilustró su exposición con estadísticas y mapas relativos al comercio de importación y exportación de cemento, tanto entre zonas de Brasil como entre países de Latinoamérica.

Entre las cuestiones tratadas en el coloquio desarrollado a continuación, el Ing. DUVOY suscitó la de la distribución de la producción de los cementos brasileños por resistencia, a la que el Ing. MENNA respondió que de las tres categorías: 400, 320 y 250, la intermedia 320 ocupaba aproximadamente el $90 \%$ de la producdión. También se hicieron algunas menciones relativas a precios.

\section{2.\%. Normalización COPANT e ISO. Sello de Conformidad}

\section{Ing. Francisco de Assis BASILIO (Brasil)}

El ponente hizo mención del hecho de que el Comité Panamericano de Normas Técnicas (COPANT) había dejado de ser en realidad panamericano, como consecuencia de la retirada del mismo por parte de los Estados Unidos. Con ello - dijo- el COPANT piende parte de su importancia al quedar reducido a su presente condición de latinoamericano. Habló después de las relaciones actuales con la RILEMI y de la necesidad de incrementarlas en el futuro. Al referirse a las especificaciones ISO-RILEM-CEMBUREAU para cementos dijo que los cementos brasileños las cumplían, así como las normas del país, y que la exportación de los cementos brasileños se podía hacer tanto de acuerdo con las mencionadas normas, como con arreglo a las ASTM o a las BS. Trató después del tema del contenido de magnesia en los cementos, problema que afecta al Brasil, y dijo que las normas ASTM habían ampliado la tolerancia en el contenido de $\mathrm{MgO}$ hasta el 6 por ciento, y que existía una propuesta de elevarla hasta el 8 por ciento. 
En el coloquio abierto a continuación se preguntó por parte de uno de los representantes uruguayos acerca del ensayo de autoclave en relación con el contenido de magnesia, a lo que el Ing. BASILIO responidió que dicho ensayo había sido puesto en tela de juicio por los norteamericanos Allan BATES y Bryant MATHER (como inciso cabría decir que también por quien esto escribe).

Acto seguido el Dr. D. VERONELLI hizo mayores precisiones sobre COPANT, comité que conoce bien como miembro que es del mismo. El Ing. BASILIO habló de la organización de la BS en el sentido de que ésta conoce y tiene fácilmente a disposición las diferencias entre las BSS y las normas de todos los demás países, de las que puede deducirse que, si bien las especificaciones son distintas, el producto (cemento) es el mismo, y que es más útil y práctico ver las cosas por el lado de las equivalencias que por el lado de las diferencias.

Refiriéndose ya al sello de calidad, el Ing. BASILIO mencionó su interés a efecto de los intercambios internacionales. En Brasil —indicó- falta la forma institucional de ponerlo en práctica. Sin embargo - dijo- para los intercambios comerciales no basta con el cumplimiento de una norma, la cual garantiza sólo la calidad de un lote aislado de la producción; es preciso un certificado que garantice la calidad y la regularidad de una amplia serie de lotes sucesivos, es decir, de una producción continuada. Esto -añadió- es del máximo interés para la comercialización internacional del cemento, y en tal sentido debería ser estudiado por el COPANT.

En el coloquio relativo al sello de calidad el Ing. BOTERO dijo que creía que el COPANT había cobrado —o debería cobrar- mayor interés para el GLAICYC, al haber dejado de pertenecer a aquél los Estados Unidos, y en consecuencia pidió para el COPANT el apoyo de las organizaciones nacionales latinoamericanas de normalización.

Hubo después otras intervenciones, entre ellas la española, en la que los Ings. GARAU y ARREDONDO hicieron breve historia del DISCAL (distintivo de calidad) español para el cemento, así como de sus vicisitudes y de su falta de aceptación. El Dr. CALLEJA explicó las razones de ello, basadas a su juicio en un error inicial en su planteamiento, ya que se estipularon condiciones drásticas muy difíciles o imposibles de cumplir, según las cuales cuanto mejor y más regular era un cemento dentro de la sujección al distintivo, mayor riesgo corría - con el DISCAL tal y como estaba establecido- de ser penalizado retirándole el distintivo de calidad. Condiciones drásticas - añadió- que eran además supérfluas.

Los representantes colombianos y argentinos manifestaron la existencia de sellos de calidad del cemento en sus respectivos países, y el Dr. VERONELLI, enlazando con la observación hecha con anterioridad por el Ing. BASILIO, habló del sello de calidad en el seno del COPANT y de las posibles directrices para su creación y desarrollo.

Tras una nueva intervención del Ing. BASILIO sobre el mismo tema, el Ing. ARREDONDO y el Dr. CALLEJA hablaron respectivamente de los DIT (Documento de Idoneidad Técnica) en general en España y en Europa -UEATC; Unión Européen pour l'Agréement Technique dans la Construction-, y de las exigencias y ensayos de control dentro de la concesión y de la vigilancia de un sello de calidad de cemento en marcha, así como de la necesidad de unas exigencias mínimas, tanto de personal formado y capacitado, como de equipo idóneo para mantener el sello con garantía de eficacia.

Representantes respectivos de Uruguay y Colombia hicieron a continuación sendas referencias a datos estadísticos y a controles de precios de cemento, en posible relación con un sello de calidad en el seno del COPANT. 
Finalmente, el Sr. COLLIS habló de los sellos de calidad del cemento en Europa, haciendo referencia al caso alemán en que existe un sello con carácter obligatorio, apoyado en las normas DIN, y al caso francés en el que existe un sello sin obligatoriedad y se apoya en las normas francesas, siendo concedido y vigilado por el Laboratorio de la Ville de París.

\subsection{Automatización y economía de combustibles}

\section{Introductor: Dr. José CALLEJA (España)}

A propuesta del Ing. PAZ, al no haber ponente de este tema, fue aceptado que hiciera la introdución al mismo y diera paso al suiosiguiente coloquio el Dr. CALLEJA.

Este comenzó recordando y ampliando más en detalle lo expuesto por él al respecto en su ponencia del día anterior, en el sentido de que a su juicio, en maquinaria y en proceso de fabricación de cemento, en cuanto a aprovechamiento y recuperación de calor se refiere, con los más modernos sistemas y equipos se había alcanzado o se estaba a punto de alcanzar prácticamente un límite mínimo de consumo térmico. Y que dentro de esta consideración se podría incluir la automatización, aún la más avanzada. La automatización - dijo- permite facilitar, regularizar y controlar el proceso y, si se quiere, también la calidad del cemento, así como rebajar el costo total del proceso y del producto. Pero -añadió- es dudoso que, dentro de todo ello, pueda reducir significativamente el consumo de combustible en el horno. El expositor llamó la atención sobre el hecho de que si la automatización regulariza el proceso y la calidad y rebaja el costo, exige a cambio condiciones a las materias primas y a los combustibles, puesto que al tenerse que ajustar a un "modelo" que impone un estrecho margen de variabilidad a los parámetros que lo definen, no puede admitir desequilibrios fuertes de dichos parámetros ni desviaciones grandes de los valores-consigna y, en consecuencia, la automatización no parece poder ser operante en procesos que utilicen como combustible carbón de baja calidad fuertemente fluctuante. Por otra parte - siguió diciendo- la automatización puede considerarse resuelta en lo que respecta a la explotación de canteras, molienda y dosificación y homogeneización de crudos, pero no tanto en cuanto al proceso de clinkerización se refiere. Y no precisamente en lo que concierne a la etapa de cocción, sino más bien - y en muy gran medida- en lo que atañe a la fase del enfriamiento del clínker. Por otro lado - añadió- ésta condicio. na de forma decisiva la marcha total del horno y en particular la de la combustión. Indicó al respecto que consideraciones mucho más detalladas sobre todos estos aspectos y muchos otros relativos a la automatización podrían encontrarse en las Memorias de los $\mathrm{V}$ Coloquios de Directores y Técnicos de Fábricas de Cemento, organizados por el IETCC y celebrados en su sede de Madrid, en 1969, publicadas en el número 138-139 de la revista "Materiales de Construcción", editada por el propio IETCC. En dichos Coloquios, que tuvieron un carácter monográfico dedicado precisamente a la automatización, el expositor —según propia manifestación - presentó un trabajo panorámico y crítico sobre el tema titulado "Consideraciones y puntos de vista generale's sobre automatización de fábricas de cemento", e hizo, con el mismo carácter, un amplio resumen con comentarios de todo lo tratado en los Coloquios, en el cual se ponía de manifiesto la complejidad del tema, a partir de la necesidad de la prehomogeneización del crudo y de las funciones de un ordenador, así como de equipos auxiliares de análisis rápidos y representativos, en los que tanto la toma automática como la preparación de las muestras juegan un papel de primer orden. A esta complejidad que parte de la base de utilizar un combustible homogéneo y prácticamente invariable, habría que añadir la nueva y grave complicación creada por el uso de carbones heterogéneos, que exigirían su propio control automático, y cuyas cenizas descorregirían el 
crudo dentro del horno, incidiendo desfavorablemente en las etapas de la automatización previas al horno. Ya se comprende - concluyó- que en tales condiciones es muy difícil pensar en una automatización eficaz, y menos aún que sea tal que permita ahorros de combustible. Finalmente el expositor indicó que, entre la documentación técnica y las publicaciones del IETCC entregadas por él a la Secretaría del GLAICYC, figuraban los trabajos mencionados

Tuvo lugar acto seguido un coloquio en el que el Dr. CENTURION hizo consideraciones sobre posibilidades de nueva maquinaria, de nuevos procesos de fabricación y de nuevas utilizaciones de otras energías — por ejemplo, la eléctrica-, en sustitución del empleo del fuel-oil y del empleo del carbón como alternativa para la fabricación de cemento. El Dr. CALLEJA señaló al respecto la utilización - siempre cara- de energía eléctrica en procesos electroquímicos tales como la obtención del aluminio, y la posibilidad de su aplicacón a la obtención futura de cementos, más o menos de tipo portland, puestó que ya era una realidad en la fabricación de cemento aluminoso (electro)-fundido, obtenido por fusión eléctrica.

El Dr. VERONELLI puntualizo e insistió sobre la influencia decisiva de la fase de enfriamiento del clínker en la automatización del proceso de fabricación del cemento, e hizo resaltar la importancia de la fácil y pronta disponibilidad de repuestos ante la eventualidad de fallos en los equipos de automatización.

En el mismo sentido se manifestó el Ing. FIERRO, añadiendo la importancia que tiene en la automatización la mano de obra idónea, y la adecuada asistencia técnica por parte de las firmas responsables.

El Ing. PAZ hizo también atinadas obervaciones en el sentido de que, dado que la automatización tenía más sentido en las fábricas de nueva planta, era preciso que el fabricante de cemento, de acuerdo con los productores de equipos, propusieran -o impusierana éstos sus condiciones, en lugar de lo contrario que, más o menos, es lo que se viene dando en la realidad actual - dijo-

\subsection{Investigación tecnológica y científica}

\section{Ing. Francisco de Assis BASILIO (Brasil)}

El ponente postuló la necesidad de un apoyo científico para la investigación en el campo de las tecnologías del cemento y del hormigón. Dijo que estas tecnologías se extendían mucho más allá del área de las consabidas - e imprescindibles- resistencias mecánicas, y que problemas tales como los relativos a la retracción (plástica, térmica y/o hidráulica), a la fisuración, a la fluencia, a la permeabilidad, (porosidad, penetrabilidad, capilaridad), al calor de hidratación, a la durabilidad, etc., tan importantes en todo tipo de obra y en particular en las grandes presas, requerían cada vez más la mayor atención. Añadió que esta base científica tan necesaria - más aún, imprescindible- escaseaba en Latinoamérica, y que era preciso, dentro de su campo, hallar el "know how" correspondiente. Puso ejemplos de problemas reales y actuales de durabilidad basados en la resistencia -y en el ataque- de hormigones de estructuras en agua de mar, así como de casos de durabilidad dependientes de la corrosión metálica de las armaduras. Destacó la importancia del ataque del aluminato tricálcico del cemento por los sulfatos, con formación de ettringita expansiva (como factor químico), así como la influencia decisiva de la relación agua/ /cemento del hormigón, a igualdad de otras consideraciones (como factor físico). Con res- 
pecto a los cementos brasileños, señaló su contenido de aluminato tricálcico comprendido entre 8 y 10 por ciento, sin problemas en cuanto a su empleo en general, incluídas en el mismo las obras marítimas y las presas.

El ponente expuso a continuación un gráfico con los resultados de unos estudios norteame ricanos de la PCA, en el que, representada por una curva la fisuración del hormigón en función del contenido de aluminato tricálcico del cemento, aquélla decrecía al aumentar éste hasta valores del orden del 10 por ciento, pese a lo cual - io por lo cual?- para obras costeras en ambiente marítimo se exigían cementos con un contenido de aluminato tricálcico del 4 al 8 por ciento.

Al iniciarse el coloquio el Ing. BASILIO recabó la participación en el mismo dei Dr. CALLEJA, el cual agradeció la invitación. Su intervención se centró fundamentalmente en dos aspectos. Uno fue el de la complejidad del ataque y de la destrucción del hormigón por agua de mar, los cuales pueden responder principalmente a causas puramente físicas como la cristalización de sales marinas (análoga a la solidificación del agua en la destrucción del hormigón por la acción del hielo), o bien a causas más complejas, como la cristalización de compuestos - yeso, ettringita- previamente formados por reacciones químicas de las sales marinas con determinados constituyentes de la pasta hidratada del cemento. El predominio de unas causas u otras -o incluso la actuación simultánea o sucesiva de ambas- depende —dijo el Dr. CALLEJA - del estado permanentemente, parcialmente o alternativamente sumergido de la obra de hormigón en el agua de mar. En tal sentido la estructura física del hormigón y de la pasta de cemento tienen - dijo- gran importancia. Además -añadió- otro motivo de complicación en el ataque del hormigón por el agua de mar es que, como ya había señalado el Ing. BASILIO, en presencia de los cloruros y de los cationes que ésta contiene, las solubilidades de los compuestos que se forman en la hidratación del cemento, o por reacción de los constituyentes de éste con las propias sales marinas, son distintas que en agua pura. de forma que las concentraciones que se alcanzan en unas condiciones dadas son también diferentes, y todo ello puede hacer variar cuantitativamente e incluso cualitativamente el curso de las reacciones, la naturaleza de los productos formados en el ataque, y las consecuencias de éste.

En el otro aspecto, el de la influencia del contenido de aluminato tricálcico del cemento, el Dr. CALLEJA dijo que habría mucho que hablar. En primer lugar, porque, además de? contenido de dicho constituyente, habría que saber en cuál o cuáles de sus posibles diversas formas cristalinas (cúbica, ortorrómbica o tetragonal), y en qué proporción de ellas se encontraba el aluminata en el cemento, pues las recientes investigaciones de Mme. M. REGOURD en el CERILH, y en la Universidad de la Sorbona de París habían puesto de relieve que no todas las varias formas cristalinas del aluminato tricálcico tenían la misma susceptibilidad frente al ataque por sulfatos y agua de mar, pareciendo ser mayor la de la forma cúbica. En segundo lugar — continuó-, porque en cualquier caso la cantidad de aluminato hidratado disponible para reaccionar expansivamente con los sulfatos del exterior dependía, a igualdad de otras cosas, del contenido de yeso del propio cemento. En tercer lugar, porque también en cualquier caso la formación de ettringita podía verificarse, o bien por reacción topoquímica entre disolución y sólido, con carácter expansivo localizado e intenso y efectos disruptivos, o por reacción a través de disolución, con cristalización en los poros y acomodación de los cristales sin ejercer presión disruptiva. También en este aspecto la estructura porosa de la pasta y la del hormigón, dependientes a su vez de la relación agua/cemento, podían ejercer una gran influencia. En cuarto lugar, porque la naturaleza primaria o secundaria de la ettringita formada podía condicionar también, en una forma u otra, el carácter expansivo o no de ésta, etc.

En cuanto a las experiencias según las cuales a mayor contenido de aluminato tricálcico en el cemento correspondía menor fisuración, el Dr. CALLEJA opinó que ello podía ser 
tan cierto como lo contrario - lo cual a primæra vista parecía más lógico-. En efecto —añadió-, todo depende (además) de la cantidad de yeso que inicialmente contenga el cemento. El yeso forma con el aluminato tricálcico los sulfoaluminatos (trisulfoaluminato y monosulfoaluminato), de los cuales el primero, la ettringita, puede ser expansivo, sobre todo si se forma con carácter topoquímico y/o secundario; y el segundo es considerado como la fase sulfoalumínica final estable en la hidratación de un aluminato tricál.. cico de un clínker. Si el cemento contiene yeso abundante, es decir, en cantidad excedente en relación con la cantidad de aluminato de su clínker, el trisulfoaluminato secundario que puede formarse puede tener un efecto expansivo que compense la retracción y por lo tanto la fisuración consiguiente. Este cemento sería de los llamados "de expansión controlada" o de "retracción compensada", y lo expuesto sucedería cualquiera que fuese su contenido de aluminato tricálcico, a condición de que la cantidad de yeso sobrepasase un mínimo necesario. Pero si el contenido de yeso no fuese el idóneo, en la hidratación no podría formarse en cantidad suficiente el trisulfoaluminato expansivo compensador de la retracción y de la fisuración, y el cemento daría pastas fisurables. Esto ocurriría asimismo cualquiera que fuese el contenido de aluminato tricálcico, del clínker, pero más si dicho contenido fuese alto y el contenido de yeso muy bajo. Por lo tanto - concluyó-, la retracción y la fisuración del cemento, por lo que respecta al aluminato tricálcico, depende más de la relación entre la cantidad de éste en el clínker y la de yeso en el cemento, que de la proporción grande o pequeña de aluminato, pudiéndose dar así, según las circunstancias, dos curvas representativas de la marcha de la fisuración en función del aluminato del cemento, las cuales podrían ser groso modo simétricas respecto de un eje normal al de los valores de aluminato, o paralelo al de los valores de fisuración. Aparte de elllo -concluyó-, también cuenta el ferrito-aluminato tetracálcico del clínker y, una vez más, la estructura porosa de la pasta del cemento y la textura y naturaleza de los poros.

En relación con esto último insistió, de acuerdo con lo señalado por el Ing. BASILIO, en la importancia de la permeabilidad o penetrabilidad del hormigón y de la pasta de cemento, tal como la tiene en cuenta el Dr. Ing. Oldrich VALENTA, y más relacionada, a su juicio, con la distribución de poros por tamaños en la pasta de cemento, resultante de las medidas con el porosímetro de mercurio según FELDMAN, que con la superficie específica de la misma, resultante de las medidas de adsorción isotérmica de nitrógeno según POWERS.

La distribución de poros por tamaños, el tamaño máximo y mínimo de los mismos, su geometría, la mayor o menor intercomunicación entre ellos, etc., pueden condicionar la penetrabilidad, la succión capilar, la mayor o menor facilidad de acomodación de los cristales que se forman o crecen, así como los efectos más o menos disruptivos de las exparsiones, o fisurantes de las retracciones, tanto como lo condicionen el contenido de aluminato tricálcico del cemento y sus formas cristalinas. Así, pues, al del efecto favorable de una menor permeabilidad debida a una menor fisuración se pueden sumar casi siempre otros factores que pueden hacer incierto o impreciso un juicio sobre la influencia aislada del contenido de aluminato tricálcico en la retracción de la pasta de cemento y en la fisuración del hormigón.

\subsection{Producción de cemento en América Latina. Datos estadísticos. Proyección. Control de precios y sus consecuencias}

En estos temas no hubo en realidad ponente, sino que se expuso por parte de cada Delegación o miembro del Grupo en forma de "rueda de mesa" coordinada por el Presidente de la Sesión. 
Aparte de las aportaciones escritas hechas a otros temas, la representación colombiana presentó a éste un documento de cuatro páginas, seis cuadros y cuatro gráficos, titulado "Informe sobre la Industria del Cemento de Colombia", firmado por el Ing. G. BOTERO, Director Ejecutivo del ICPC.

Los delegados de cada país miembro del Grupo expusieron por turno los datos estadísticos relativos a capacidades, producciones, exportaciones, importaciones, regulaciones de la producción, el consumo y el mercado interior de cada país, etc. Las situaciones presentadas fueron bastante distintas, sobre todo en cuanto a precios, al control o libertad de los mismos y al hecho de ser iguales para toda la producción o distintos según las fábricas (como es el caso de Colombia).

Al final de la exposición el Dr. CALLEJA hizo una propuesta en el sentido de que la Secretaría General del Grupo, con los datos y sugerencias necesarios, recabados de los miembros del mismo, elaborase un esquema o cuadro de presentación de datos estadísticos, para uso común de dichos miembros, con objeto de hacer más fácilmente comparables en lo sucesivo los datos de los distintos países.

\subsection{Cuestiones pendientes}

Se trató bajo este título del tema de los coordinadores regionales como delegados permanentes del Secretario General del GLAICYC, asunto planteado como continuación del Acta de Medellín (ver 2.1.).

Se convino en estudiar el tema con más detenimiento, para replantearlo en la próxima reunión plenaria del Grupo.

\section{CONCLUSIONES Y CLAUSURA}

Entre los debates suscitados a la hora de fijar las conclusiones figuró en primer lugar el relativo al nombre de la entidad asociativa de las instituciones, dado que se han venido utilizando indistintamente las denominaciones de "Reunión" y de "Grupo". Tras una serie de matizaciones y precisiones al respecto se tomó el acuerdo de llamarla GRUPO (Latino-americano de Instituciones del Cemento y del Concreto) y de designarla con la sigla GLAICYC.

Se suscitó también la cuestión de si convenía o no de dar al Grupo un carácter más formalista que el que hasta ahora ha venido teniendo. En relación con ello se hicieron diversas consideraciones y se manifestaron varias opiniones acerca de la misión de coopera ción dentro del GLAICYC, de la conveniencia de que dentro del mismo tuvieran acogida los sectores cementero y constructor de los países miembros, de la necesidad de que el grupo tomase más en consideración los aspectos técnicos-económicos relativos al cemento, y de la oportunidad de crear dentro del GLAICYC Secciones y Sub-secciones "ad hoc" para tratar temas específicos según lo requiriesen las circunstancias.

Por acuerdo, mayoritario en principio y unánime después, se convino en no cambiar el carácter ágil del Grupo, ni sujetarlo a formalidades que pudieran coartar su libertad de actuación con trabas administrativas, financieras o de otra índole.

Se acordó también por unanimidad que la Secretaría General del GLAICYC continuase en Medellín (Colombia) y se aceptó por aclamación la propuesta de que constase en acta 
el agradecimiento y la felicitación de todas las Instituciones miembros al Secretario, General saliente, Ing. Jorge Ignacio PAZ PARRA, por su labor eficaz y ejemplar al frente de la Secretaría durante los seis años de su actuación como tal. Asimismo se acordó que constase en acta la bienvenida del Ing. Germán BOTERO ARANGO como nuevo Secretario General del Grupo, por parte de los miembros del mismo, con la felicitación de éstos por su nombramiento para el cargo.

Se acordó igualmente que la próxima Reunión Plenaria del Grupo tuviera lugar, dentro de tres años, en algún lugar de la Subregión Norte.

Finalmente se aceptó y agradeció el ofrecimiento argentino para que la próxima reunión subregional tuviera lugar en Buenos Aires, dentro de un año.

Agotado el programa y sin más asuntos que tratar, el Presidente dió por clausurada la 2. ${ }^{a}$ Reunión del Grupo Latinoamericano de Instituciones del Cemento y del Concreto.

\section{VISITAS TECNICAS}

Después de las reuniones del Grupo los miembros de éste realizaron dos visitas técnicas. Una a las obras en construcción del futuro Instituto Brasileño de Metrología, cuya misión será la de resolver los problemas metrológicos que la industria le pueda plantear en los campos de la mecánica, de la electricidad, de la electrónica, de la acústica, etc.

La otra visita efectuada fue a las instalaciones, sistemas y equipos de información y control del aeropuerto internacional de Galeao (Río de Janeiro) de la Isla del Gobernador, en la Bahía de Guanabara.

\section{OTROS ACTOS}

Sin protocolo ni ceremonial, pero con el calor de la amistad, durante las reuniones del Grupo les fueron entregadas a las Instituciones de los países miembros del mismo sendas medallas conmemorativas del 50 Aniversario (1929-1979) de la Revista Técnica española CEMENTO-HORMIGON, tan conocida, difundida y prestigiada en Latinoamérica.

La entrega, en nombre de Don Patricio PALOMAR COLLADO, fundador y director de la Revista y de Don Patricio y Don Carlos PALOMAR LLOVET, redactor-jefe de la misma y director de la editorial "Editores Técnicos Asociados" de Barcelona (España), respectivamente, fue hecha por el Ing. Ernesto GARAU, Presidente de la Agrupación de Fabricantes de Cemento de España.

Entre la documentación técnica aportada por el Dr. José CALLEJA al fondo bibliográfico de la Secretaría General del GLAICYC (véase 2.8.), figura un ejemplar del número extraordinario 546 de la Revista Técnica CEMENTO-HORMIGON, conmemorativo del Cincuentenario de la misma. Como dato oportuno en esta ocasión se recuerda aquí que en dicho número figuran las opiniones de algunas Instituciones latino-americanas acerca de la Revista. Entre ellas las del Instituto del Cemento Portland Argentino (Ing. Ernesto DUVOY, Director Técnico), el Instituto Chileno del Cemento (Ing. Eduardo GOMIEN, Director Gerente), el Instituto Colombiano de productores de Cemento (Ing. Jorge Ignacio PAZ P., Director Ejecutivo), el Instituto Mexicano del Cemento y del Concreto (Ing. Cutberto DIAZ GOMEZ, Director), la Asociación de Fabricantes de Cemento Portland del Uruguay (Ing. Oscar PAROLI, Presidente y Dr. Gustavo VAZQUEZ FIOL, Secretario), la Asociación Brasileña de Cemento Portland (Ing. Francisco de Assis BASILIO, Presidente), y la Secretaría del Grupo Latinoamericano de Instituciones del Cemento y del Concreto (Ing. Jorge Ignacio PAZ P., Secretario General). 